\title{
COMPUTING GRÖBNER BASES AND INVARIANTS OF THE SYMMETRIC ALGEBRA
}

\author{
M. LA BARBIERA AND G. RESTUCCIA
}

Received 09 September, 2014

\begin{abstract}
We study algebraic invariants of the symmetric algebra $\operatorname{Sym}_{R}(L)$ of the square-free monomial ideal $L=I_{n-1}+J_{n-1}$ in the polynomial ring $R=K\left[X_{1}, \ldots, X_{n} ; Y_{1}, \ldots, Y_{n}\right]$, where $I_{n-1}$ (resp. $\left.J_{n-1}\right)$ is generated by all square-free monomials of degree $n-1$ in the variables $X_{1}, \ldots, X_{n}$ (resp. $Y_{1}, \ldots, Y_{n}$ ). In particular, the dimension and the depth of $\operatorname{Sym}_{R}(L)$ are investigated by techniques of Gröbner bases and theory of $s$-sequences.
\end{abstract}

2010 Mathematics Subject Classification: 13A99; 13C15; 13P10

Keywords: Gröbner bases, symmetric algebra, dimension, depth

\section{INTRODUCTION}

Let $R$ be a commutative noetherian ring and $M$ be a finitely generated $R$-module $M=R f_{1}+\cdots+R f_{q}$. If $\left(a_{i j}\right), i=1, \ldots, q, j=1, \ldots, p$, is the matrix associated to a free presentation of $M$, then $\operatorname{Sym}_{R}(M)=R\left[T_{1}, \ldots, T_{q}\right] / J$, where $J$ is generated by the linear forms $g_{j}=\sum_{i=1}^{q} a_{i j} T_{i}$ for $j=1, \ldots, p$.

In [3], in order to study the symmetric algebra $\operatorname{Sym}_{R}(M)$, it is introduced the concept of $s$-sequence for the generators $f_{1}, \ldots, f_{q}$ of $M$. We say that $f_{1}, \ldots, f_{q}$ is an $s$-sequence for $M$ if there exists a monomial order $\prec$ for the monomials in the variables $T_{i}$ with $T_{1} \prec T_{2} \prec \cdots \prec T_{q}$ such that $i n_{\prec}(J)=\left(\ell_{1} T_{1}, \ldots, \ell_{q} T_{q}\right)$, with $d_{i}=\left(f_{1}, \ldots, f_{i-1}\right): R f_{i}$ ideals of $R$.

The ideals $I_{i}$ are called the annihilator ideals of the sequence $f_{1}, \ldots, f_{q}$. If $M$ is generated by an $s$-sequence, the standard algebraic invariants of $M$ can be expressed only by the ideals $d_{i}$ and in more cases the dimension can be computed in terms of the annihilators ideal of the sequence. The crucial point is that we can easily calculate the invariants, starting by the structure of the initial ideal $i n_{\prec}(J)$ of $J$, stated that it is $\underline{T}$-linear, being $\underline{T}=\left\{T_{1}, \ldots, T_{q}\right\}$ the variables that correspond in the presentation of symmetric algebra of $M$ to the generators of $M$. A natural question arises: if $i n_{\prec}(J)$ is not linearly generated in the variables $T_{1}, \ldots, T_{q}$ and we write $i n_{\prec}(J)=J_{L}+J^{*}$, where $J^{*}$ is the not linear part of $i n_{\prec}(J)$, what is the maximum degree (with respect the variables $T_{1}, \ldots, T_{q}$ ) of the monomial generators of $J^{*}$ ? 
We are interested to check when $J^{*}$ is generated by monomials of minimum degree (quadratic). Moreover, we are interested to describe explicitly a Gröbner basis of $J$, given the importance of the initial ideal in more open problems about the invariants of $\operatorname{Sym}_{R}(M)$ ([2], [4], [5], [8]).

Now, let $R=K\left[X_{1}, \ldots, X_{n} ; Y_{1}, \ldots, Y_{m}\right]$ be the polynomial ring on a field $K$ in two sets of variables and $L$ be a mixed products ideal, as defined in [7]. In [6] the authors selected the mixed products ideals that are generated by an $s$-sequence in order to compute the value or a bound for standard invariants of the symmetric algebra $\operatorname{Sym}_{R}(L)$. The problem is open for ideals $L$ that are not generated by an $s$-sequence. In this paper we examine a first class of mixed products ideals not generated by an $s$-sequence. More precisely, we consider the ideal $L=I_{n-1}+J_{n-1}$ of $K\left[X_{1}, \ldots, X_{n} ; Y_{1}, \ldots, Y_{n}\right]$, with $I_{n-1}$ (resp. $\left.J_{n-1}\right)$ the monomial ideal of $R$ generated by all square-free monomials of degree $n-1$ in the variables $X_{1}, \ldots, X_{n}$ (resp. $\left.Y_{1}, \ldots, Y_{n}\right)$.

The aim is to compute a Gröbner basis of the relations ideal $J$ of the symmetric algebra $\operatorname{Sym}_{R}(L)$ and to study some invariants of $\operatorname{Sym}_{R}(L)$. More precisely, in section 1 we give the structure of a Gröbner basis of $J$ with respect to the lexicographic order $\prec$. It should be noted that we are able to compute the generators of $i_{\prec}(J)$ not linear in the variables $T_{1} \ldots, T_{2 n}$ and to establish the degree. For $n=m=3$, we obtain the only case in which the not linear part of $i n_{\prec}(J)$ is of degree two in the variables $T_{i}$. In section 2, we compute the dimension and the depth of $\operatorname{Sym}_{R}(L)$. For the computation of the dimension, we inspire to the techniques used in the theory of the $s$-sequences. More precisely, we consider the linear part $J_{L}$ of $i n_{\prec}(J)$ and we apply the results given in [3] for computing dimension in terms of the annihilator ideals of the monomial sequence generating $L$. Then we obtain that $\operatorname{Sym}_{R}(L)$ is a Cohen-Macaulay algebra.

\section{GRÖBNER BASES OF RELATION IDEALS}

In [3] the notion of $s$-sequence is introduced for finitely generated modules in a noetherian ring $R$.

For every $i=1, \ldots, q$, we set $M_{i-1}=R f_{1}+\cdots+R f_{i-1}$ and $d_{i}=M_{i-1}:_{R} f_{i}$ be the colon ideal. We set $d_{0}=(0)$. Since $M_{i} / M_{i-1} \simeq R / d_{i}$, so $d_{i}$ is the annihilator of the cyclic module $R / d_{i} . d_{i}$ is called annihilator ideal of the sequence $f_{1}, \ldots, f_{q}$.

Let $\left(a_{i j}\right)$, for $i=1, \ldots, q, j=1, \ldots, p$, be the relation matrix of $M$. The symmetric algebra $\operatorname{Sym}_{R}(M)$ has a presentation $R\left[T_{1}, \ldots, T_{q}\right] / J$, with $J=\left(g_{1}, \ldots, g_{p}\right)$ where $g_{j}=\sum_{i=1}^{q} a_{i j} T_{i}$ for $j=1, \ldots, p$.

We consider $S=R\left[T_{1}, \ldots, T_{q}\right]$ as a graded ring by assigning to each variable $T_{i}$ degree 1 and to the elements of $R$ degree 0 .

Let $\prec$ be a monomial order on the monomials of $S$ in the variables $T_{i}$ such that $T_{1} \prec T_{2} \prec \cdots \prec T_{q}$. With respect to this term order, if $f=\sum a_{\alpha} \underline{T}^{\alpha}$, where $\underline{T}^{\alpha}=$ 
$T_{1}^{\alpha_{1}} \cdots T_{q}^{\alpha_{q}}$ and $\alpha=\left(\alpha_{1}, \ldots, \alpha_{q}\right) \in \mathbb{N}^{q}$, we put $i_{\prec}(f)=a_{\alpha} \underline{T}^{\alpha}$, where $\underline{T}^{\alpha}$ is the largest monomial in $f$ such that $a_{\alpha} \neq 0$.

So we define the monomial ideal $\operatorname{in}_{\prec}(J)=\left(\left\{i_{\prec}(f) \mid f \in J\right\}\right)$. In general we have $\left(d_{1} T_{1}, d_{2} T_{2}, \ldots, d_{q} T_{q}\right) \subseteq$ in $(J)$ and the two ideals coincide in degree 1 .

The sequence $f_{1}, \ldots, f_{q}$ is an $s$-sequence for $M$ if

$$
\left(d_{1} T_{1}, \ell_{2} T_{2}, \ldots, \ell_{q} T_{q}\right)=i n_{\prec}(J) .
$$

If $R$ is a polynomial ring over a field and $f_{1}, \ldots, f_{q}$ are monomials of $R$, then we have a criterion to be $s$-sequences. Set $f_{i j}=\frac{f_{i}}{\left[f_{i}, f_{j}\right]}$ for $i \neq j$, where $\left[f_{i}, f_{j}\right]$ is the greatest common divisor of the monomials $f_{i}$ and $f_{j}$. $J$ is generated by $g_{i j}=$ $f_{i j} T_{j}-f_{j i} T_{i}$ for $1 \leq i<j \leq q$. The monomial sequence $f_{1}, \ldots, f_{q}$ is an $s$-sequence if and only if $g_{i j}$, for $1 \leq i<j \leq q$, is a Gröbner basis for $J$ for a term order that agrees with the order of the variables in $S=R\left[T_{1}, \ldots, T_{q}\right]$. Note that the annihilator ideals of the monomial sequence $f_{1}, \ldots, f_{q}$ are the ideals $d_{i}=\left(f_{1 i}, f_{2 i}, \ldots, f_{i-1, i}\right)$ for $i=1, \ldots, q([3])$.

Now we consider the polynomial ring $R=K\left[X_{1}, \ldots, X_{n} ; Y_{1}, \ldots, Y_{m}\right]$ over a field $K$ in two sets of variables and the class of monomial ideals of mixed products of $R=K\left[X_{1}, \ldots, X_{n} ; Y_{1}, \ldots, Y_{m}\right]$ :

$$
L=I_{k} J_{r}+I_{s} J_{t},
$$

where $I_{k}$ (resp. $J_{r}$ ) is the monomial ideal of $R$ generated by all the square-free monomials of degree $k$ (resp. $r$ ) in the variables $X_{1}, \ldots, X_{n}$ (resp. $Y_{1}, \ldots, Y_{m}$ ).

In [6] the authors investigate in which cases these monomial ideals are generated by an $s$-sequence. The system of generators of $L$ is an $s$-sequence only in the following cases:

1) $L=I_{n-1} J_{m}$, 2) $L=I_{1} J_{m}$, 3) $L=I_{n-1} J_{m}+I_{n} J_{m-1}$, 4) $L=J_{m}+I_{n} J_{1}$.

Set $I_{0}=J_{0}=R$, then we have the following case for $r=0$ and $s=0 L=I_{k}+J_{k}$ with $1 \leq k \leq \inf \{n, m\}$. We study this class of square-free monomial ideals for $k=$ $n-1$ and $n=m$, then $R=K\left[X_{1}, \ldots, X_{n} ; Y_{1}, \ldots, Y_{n}\right]$ and $L=I_{n-1}+J_{n-1}$.

Since the property to be an $s$-sequence may depend on the order of the sequence, in the sequel we will suppose $L=\left(f_{1}, f_{2}, \ldots, f_{2 n}\right)$ where $f_{1} \prec f_{2} \prec \cdots \prec f_{2 n}$ with respect to the monomial order $\prec_{\text {lex }}$ on $X_{1}, \ldots, X_{n}, Y_{1}, \ldots, Y_{n}$ and $X_{1} \prec X_{2} \prec \cdots \prec$ $X_{n} \prec Y_{1} \prec Y_{2} \prec \cdots \prec Y_{n}$.

$J$ is generated by $g_{i j}=f_{i j} T_{j}-f_{j i} T_{i}$ for $1 \leq i<j \leq 2 n$. The monomial sequence $f_{1}, \ldots, f_{2 n}$ is an $s$-sequence if and only if $g_{i j}$ for $1 \leq i<j \leq 2 n$ is a Gröbner basis for $J$ in $K\left[X_{1}, \ldots, X_{n} ; Y_{1}, \ldots, Y_{n} ; T_{1}, \ldots, T_{2 n}\right]$, with $X_{1} \prec X_{2} \prec \cdots \prec X_{n} \prec Y_{1} \prec$ $Y_{2} \prec \cdots \prec Y_{n} \prec T_{1} \prec T_{2} \prec \cdots \prec T_{2 n}$.

Theorem 1 ([6]). Let $R=K\left[X_{1}, \ldots, X_{n}\right]$ be the polynomial ring over a field $K$ and $I_{k}$ with $2 \leq k \leq n$. The ideal $I_{k}$ is generated by an s-sequence if and only if $k=n-1$. 
Theorem 2. Let $R=K\left[X_{1}, \ldots, X_{n} ; Y_{1}, \ldots, Y_{n}\right]$ be the polynomial ring over a field $K$ and $L=I_{n-1}+J_{n-1}$. $L$ is not generated by an s-sequence for any $n \neq 2$.

Proof. For $n=2, L=I_{1}+J_{1}=\left(X_{1}, \ldots, X_{n}, Y_{1}, \ldots, Y_{n}\right)$ is generated by an $s$ sequence for any admissible term order, since $L$ is generated by a regular sequence ([3]).

For $n>2$, let $L=I_{n-1}+J_{n-1}=\left(f_{1}, f_{2}, \ldots, f_{n}\right)+\left(f_{n+1}, f_{n+2}, \ldots, f_{2 n}\right)$, where $f_{1} \prec f_{2} \prec \cdots \prec f_{n}$ and $f_{n+1} \prec f_{n+2} \prec \cdots \prec f_{2 n}$ with respect to the monomial order $\prec_{l e x} . L$ is generated by an $s$-sequence $\Leftrightarrow G=\left\{g_{i j}=f_{i j} T_{j}-f_{j i} T_{i} \mid 1 \leq i<j \leq\right.$ $2 n\}$ is a Gröbner basis for $J \Leftrightarrow S\left(g_{i j}, g_{h l}\right) \stackrel{G}{\rightarrow} 0$ for all $i, j, h, l \in\{1, \ldots, 2 n\}$ and $g_{i j} \neq g_{h l}$. We consider a lexicographic Gröbner basis for $J$ with respect to the order on the variables $T_{1} \prec T_{2} \prec \cdots \prec T_{2 n}$.

The generators of $L$ are the following: $f_{1}=X_{1} \cdots X_{n-1}, f_{2}=X_{1} \cdots X_{n-2} X_{n}$, $f_{n}=X_{2} \cdots X_{n}, f_{n+1}=Y_{1} \cdots Y_{n-1}, f_{n+2}=Y_{1} \cdots Y_{n-2} Y_{n}, \ldots, f_{2 n}=Y_{2} \cdots Y_{n}$.

One has $S\left(g_{1 n}, g_{2, n+1}\right)=\frac{f_{1 n} f_{n+1,2}}{\left[f_{1 n}, f_{2, n+1}\right]} T_{2} T_{n}-\frac{f_{2, n+1} f_{n 1}}{\left[f_{1 n}, f_{2, n+1}\right]} T_{1} T_{n+1}=$ $Y_{1} \cdots Y_{n-1} T_{2} T_{n}-X_{2} \cdots X_{n-2} X_{n}^{2} T_{1} T_{n+1}$. By the structure of the generators of $L$ there is no $g_{s t} \in G$ whose initial term with respect to the admissible order on the variables $T_{1} \prec T_{2} \prec \cdots \prec T_{2 n}$ divides the terms of $S\left(g_{1 n}, g_{2, n+1}\right)$. It follows that it is not possible to get a standard expression of $S\left(g_{1 n}, g_{2, n+1}\right)$ with respect $G$ with remainder 0 . Hence $G$ is not a Gröbner basis for $J$. It follows that $L$ can not be generated by an $s$-sequence ([3], Lemma 1.2). In fact, from the theory of Gröbner bases, suppose that $f_{1}, \ldots, f_{2 n}$ is a monomial $s$-sequence with respect to some admissible term order $\prec$, then $f_{1}, \ldots, f_{2 n}$ is an $s$-sequence for any other admissible term order, and as a consequence it is an $s$-sequence for the lexicographic order, that is a contradiction.

The main result of this section gives the structure of the Gröbner basis of the relation ideal $J$ of the symmetric algebra $\operatorname{Sym}_{R}(L)$.

Theorem 3. Let $R=K\left[X_{1}, \ldots, X_{n} ; Y_{1}, \ldots, Y_{n}\right]$ be the polynomial ring over a field $K$ and $L=I_{n-1}+J_{n-1}$. A Gröbner basis for $J$ is the set

$$
B G(J)=\left\{g_{i j}=f_{i j} T_{j}-f_{j i} T_{i}, 1 \leq i<j \leq 2 n\right\} \cup 8,
$$

where $8=\bigcup_{t=1}^{n-2} H_{t}$ with $H_{1}=\bigcup_{k_{1}=3}^{n} H_{1 k_{1}}$ where $H_{1 k_{1}}=\left\{S\left(g_{1 k_{1}}, g_{i j}\right) \mid i<i<\right.$ $\left.k_{1}, \quad j=n+1, \ldots, 2 n\right\}$ and $H_{t}=\bigcup_{k_{t}=t+2}^{n} H_{t k_{t}}$ where $H_{t k_{t}}=\left\{S\left(g_{1 k_{t}}, h\right) \mid h \in\right.$ $\left.H_{t-1 k_{t-1}}, k_{t-1}<k_{t}\right\}$.

Proof. Let $L=I_{n-1}+J_{n-1}=\left(f_{1}, \ldots, f_{n}\right)+\left(f_{n+1} \ldots, f_{2 n}\right)$ and $G=\left\{g_{i j}=\right.$ $\left.f_{i j} T_{j}-f_{j i} T_{i} \mid 1 \leq i<j \leq 2 n\right\}$, where $f_{i j}=\frac{f_{i}}{\left[f_{i}, f_{j}\right]}$ for $i \neq j$ and $\left[f_{i}, f_{j}\right]$ is the greatest common divisor of the monomials $f_{i}$ and $f_{j}$. By the Theorem $2 L$ is not generated by an $s$-sequence, then $G \varsubsetneqq B G(J)$ and there are $S$-polynomials $S\left(g_{i j}, g_{k l}\right)$ that have not a standard expression with respect $G$ with remainder 0 . Let $g_{i j}, g_{k l} \in G$, 
it is known that $S\left(g_{i j}, g_{k l}\right)=\frac{f_{i j} f_{l k}}{\left[f_{i j}, f_{k l}\right]} T_{j} T_{k}-\frac{f_{k l} f_{j i}}{\left[f_{i j}, f_{k l}\right]} T_{i} T_{l}$. Knowing the structure of the monomials $f_{1}, \ldots, f_{2 n}$, we are able to compute the $S$-polynomials of $G$ that do not reduce to 0 modulo $G$ and using Buchberger algorithm we construct the elements of $B G(J) \backslash G$. Then for $j=n+1, \ldots, 2 n$ we compute $S\left(g_{1 k_{1}}, g_{i j}\right)$ with $i<k_{1}$ and $k_{1}=3, \ldots, n$ :

$$
\begin{aligned}
& S\left(g_{13}, g_{2 j}\right)=f_{j} T_{2} T_{3}-\delta_{1} X_{n}^{2} T_{1} T_{j} \\
& S\left(g_{14}, g_{2 j}\right)=f_{j} T_{2} T_{4}-\delta_{2} X_{n}^{2} T_{1} T_{j} \\
& S\left(g_{15}, g_{2 j}\right)=f_{j} T_{2} T_{5}-\delta_{4} X_{n}^{2} T_{1} T_{j}
\end{aligned}
$$

$$
S\left(g_{1 n}, g_{n-1, n+1}\right)=f_{j} T_{n-1} T_{n}-\delta_{\left(\begin{array}{c}
n-1 \\
n-3
\end{array}\right)} X_{n}^{2} T_{1} T_{j},
$$

where $\delta_{1}, \delta_{2}, \ldots, \delta_{\left(\begin{array}{c}n-1 \\ n-3\end{array}\right)}$ are the generators of the Veronese ideal of degree $n-3$ in the variables $X_{1}, X_{2}, \ldots, X_{n-1}$. Being $f_{1}=X_{1} \cdots X_{n-1}, f_{2}=X_{1} \cdots X_{n-2} X_{n}, f_{n}=$ $X_{2} \cdots X_{n}, f_{n+1}=Y_{1} \cdots Y_{n-1}, f_{n+2}=Y_{1} \cdots Y_{n-2} Y_{n}, \ldots, f_{2 n}=Y_{2} \cdots Y_{n}$, it follows that there is no $g_{s t} \in G$ whose initial term divides the terms of these $S$-polynomials. Then $S\left(g_{1 k_{1}}, g_{i j}\right) \in B G(J)$ for $j=n+1, \ldots, 2 n, i<k_{1}$ and $k_{1}=3, \ldots, n$. Set $H_{1 k_{1}}=\left\{S\left(g_{1 k_{1}}, g_{i j}\right) \mid j=n+1, \ldots, 2 n, i<k_{1}\right\}$ for $k_{1}=3, \ldots, n$. Now we continue to compute the $S$-polynomials $S\left(g_{1 k_{2}}, S\left(g_{1 k_{1}}, g_{i j}\right)\right)$ with $i<k_{1}<k_{2}$ and $k_{2}=4, \ldots, n, j=n+1, \ldots, 2 n$ :

$$
\begin{aligned}
& S\left(g_{14}, S\left(g_{13}, g_{2, n+1}\right)\right)=f_{n+1} T_{2} T_{3} T_{4}-\gamma_{1} X_{n}^{3} T_{1}^{2} T_{n+1} \\
& S\left(g_{14}, S\left(g_{13}, g_{2, n+2}\right)\right)=f_{n+2} T_{2} T_{3} T_{4}-\gamma_{2} X_{n}^{3} T_{1}^{2} T_{n+2}
\end{aligned}
$$

$$
S\left(g_{14}, S\left(g_{13}, g_{2,2 n}\right)\right)=f_{2 n} T_{2} T_{3} T_{4}-\gamma_{\left(\begin{array}{c}
n-1 \\
n-4
\end{array}\right)} X_{n}^{3} T_{1}^{2} T_{2 n}
$$

and so on up to $k_{2}=n$ :

$$
\begin{aligned}
& S\left(g_{1 n}, S\left(g_{1, n-1}, g_{n-2, n+1}\right)\right)=f_{n+1} T_{n-2} T_{n-1} T_{n}-\gamma_{1} X_{n}^{3} T_{1}^{2} T_{n+1} \\
& S\left(g_{1 n}, S\left(g_{1, n-1}, g_{n-2, n+2}\right)\right)=f_{n+2} T_{n-2} T_{n-1} T_{n}-\gamma_{2} X_{n}^{3} T_{1}^{2} T_{n+2}
\end{aligned}
$$




$$
S\left(g_{1 n}, S\left(g_{1, n-1}, g_{n-2,2 n}\right)\right)=f_{2 n} T_{n-2} T_{n-1} T_{n}-\gamma_{\left(\begin{array}{c}
n-1 \\
n-4
\end{array}\right)} X_{n}^{3} T_{1}^{2} T_{2 n},
$$

where $\gamma_{1}, \gamma_{2}, \ldots, \gamma_{\left(\begin{array}{c}n-1 \\ n-4\end{array}\right)}$ are the generators of the Veronese ideal of degree $n-4$ in the variables $X_{1}, X_{2}, \ldots, X_{n-1}$. The terms of these $S$-polynomials are not divided by the initial term of any $g_{s t} \in G$, then $S\left(g_{1 k_{2}}, S\left(g_{1 k_{1}}, g_{i j}\right)\right) \in B G(J)$ for $i<k_{1}<k_{2}$ and $k_{2}=4, \ldots, n, j=n+1, \ldots, 2 n$. Set $H_{2 k_{2}}=\left\{S\left(g_{1 k_{2}}, h_{k_{1}}\right) \mid h_{k_{1}} \in H_{1 k_{1}}\right\}$. Continuing the computation of the $S$-polynomials one obtains:

$$
H_{3 k_{3}}=\left\{S\left(g_{1 k_{3}}, h_{k_{2}}\right) \mid h_{k_{2}} \in H_{2 k_{2}}\right\} \text { for } k_{2}<k_{3}, k_{3}=5, \ldots, n
$$

$$
\begin{aligned}
\begin{array}{r}
H_{n-3 k_{n-3}} \\
n-1, n
\end{array} & \left\{S\left(g_{1 k_{n-3}}, h_{k_{n-4}}\right) \mid h_{k_{n-4}} \in H_{n-4 k_{n-4}}\right\} \text { for } k_{n-4}<k_{n-3}, k_{n-3}= \\
H_{n-2 k_{n-2}} & =\left\{S\left(g_{1 k_{n-2}}, h_{k_{n-3}}\right) \mid h_{k_{n-3}} \in H_{n-3 k_{n-3}}\right\} \text { for } k_{n-3}<k_{n-2}, k_{n-2}=n .
\end{aligned}
$$

Set $8=\bigcup_{t=1}^{n-2} H_{t}$ with $H_{1}=\bigcup_{k_{1}=3}^{n} H_{1 k_{1}}$ where $H_{1 k_{1}}=\left\{S\left(g_{1 k_{1}}, g_{i j}\right) \mid i<i<\right.$ $\left.k_{1}, \quad j=n+1, \ldots, 2 n\right\}$ and $H_{t}=\bigcup_{k_{t}=t+2}^{n} H_{t k_{t}}$ where $H_{t k_{t}}=\left\{S\left(g_{1 k_{t}}, h\right) \mid h \in\right.$ $\left.H_{t-1 k_{t-1}}, k_{t-1}<k_{t}\right\}$. Then elements of $\delta$ do not reduce to 0 modulo $G$. Moreover by construction no term of an element $h$ of $\delta$ is divisible by the initial term of an element of $\delta \backslash\{h\}$. Set $B=G \cup \delta$, it follows that $B G(J) \supseteq B$.

In order to show that $B G(J)=B$ we must prove that for all $g, h \in B$ the $S$ polynomial $S(g, h)$ reduces to 0 modulo $B$.

Let's start to prove that all the $S$-polynomials $S\left(g_{i j}, g_{h l}\right)$, with $i, j, h, l \in\{1, \ldots, 2 n\}$, has a standard expression with respect to $B$ with remainder 0 . We have:

$$
S\left(g_{i j}, g_{h l}\right)=\frac{f_{i j} f_{l h}}{\left[f_{i j}, f_{h l}\right]} T_{j} T_{h}-\frac{f_{h l} f_{j i}}{\left[f_{i j}, f_{h l}\right]} T_{i} T_{l} .(*)
$$

Let's find a standard expression of $S\left(g_{i j}, g_{h l}\right)$, for all $i, j, h, l \in\{1, \ldots, n-1\}$.

If $\left[i n_{\prec}\left(g_{i j}\right), i n_{\prec}\left(g_{h l}\right)\right]=1$, then $S\left(g_{i j}, g_{h l}\right)=f_{l h} g_{i j} T_{h}-f_{j i} g_{h l} T_{i}$.

If $\left[i n_{\prec}\left(g_{i j}\right), i n_{\prec}\left(g_{h l}\right)\right] \neq 1$, we apply $(*)$ to obtain a standard expression for the $S$-polynomials $S\left(g_{i j}, g_{h l}\right)$. It results:

$$
\begin{aligned}
& S\left(g_{i j}, g_{i l}\right)=-\left[f_{j i}, f_{l i}\right] g_{j l} T_{i} \\
& S\left(g_{i j}, g_{l j}\right)=\left[f_{j i}, f_{j l}\right] g_{i l} T_{j} \\
& S\left(g_{i j}, g_{l k}\right)=\left[f_{j i}, f_{k l}\right]\left(\frac{f_{k l}}{\left[f_{j i}, f_{k l}\right]} g_{i l} T_{j}-\frac{f_{l k}}{\left[f_{i j}, f_{l k}\right]} g_{j k} T_{i}\right) \text { or } \\
& S\left(g_{i j}, g_{l k}\right)=\left[f_{j i}, f_{k l}\right]\left(\frac{f_{j i}}{\left[f_{j i}, f_{k l}\right]} g_{i l} T_{k}-\frac{f_{i j}}{\left[f_{i j}, f_{l k}\right]} g_{j k} T_{l}\right) .
\end{aligned}
$$

Hence all the $S$-polynomials $S\left(g_{i j}, g_{h l}\right)$ reduce to 0 with respect to $B$. It remains to prove that the elements of $B \backslash G$ reduce to 0 with respect to $B$. If the elements 
of $B$ have initial terms coprime, then they reduce to 0 with respect to $B$. Otherwise we observe that by the structure of the elements of $\delta$ it follows that the initial terms of the elements of $\delta$ are $\gamma_{i_{k}} X_{n}^{t} T_{1}^{s} T_{j}$ with $\gamma_{i_{k}}$ a generator of the Veronese ideal of degree $k$ in the variables $X_{1}, X_{2}, \ldots, X_{n-1}$ for $n+1 \leq j \leq 2 n, 0 \leq k \leq n-3,0 \leq$ $t \leq n-1,0 \leq s \leq n-3, k+t=n-1, s=t-1$.

Let $f, g \in B$, let $d$ be the greater common divisor of in in $_{\prec}(f)$ and in $\prec(g), c$ be the greater common divisor of the no initial terms of $f$ and $g$. In order to prove that $S(f, g)$ reduces to 0 with respect to $B$ for all $f, g \in B$, we start to consider the elements of $B$ of the form $g_{i j} \in G$ and $g_{1 m, s t}=S\left(g_{1 m}, g_{s t}\right) \in H_{1 k_{1}}$. We have the following cases:

- if $T_{j}$ is a variable of $d$, then $S\left(g_{i j}, g_{1 m, s t}\right)$ reduces to 0 by the elements of $B$ $g_{i s}$ and $g_{1 m}$ for $i<s$;

- if $T_{j}$ is a variable of $d$ and $T_{i}$ is a variable of $c$, then $S\left(g_{i j}, g_{1 j, s i}\right)$ reduces to 0 by the element $g_{1 s} \in B$;

- if no variable $T_{1}, \ldots, T_{2 n}$ is in $d$ and $c$, then $S\left(g_{i j}, g_{1 m, s t}\right)$ reduces to 0 by the elements of $B g_{1 i}$ and $g_{11 m, s t j}=S\left(g_{1 j}, S\left(g_{1 t}, g_{s m}\right)\right) \in H_{2 k_{2}}$ for $s<t<j$.

For the elements of the form $g_{1 j, l k}=S\left(g_{1 j}, g_{l k}\right) \in G$ and $g_{1 m, s t}=S\left(g_{1 m}, g_{s t}\right) \in$ $H_{1 k_{1}}$, we have the following cases:

- if $T_{1}, T_{j}$ are variables of $d$ and $T_{l}$ is a variable of $c$, then $S\left(g_{1 j, l k}, g_{1 j, t l}\right)$ reduces to 0 by $g_{k t} \in B$ for $k<t$;

- if $T_{1}$ is a variable of $d$ and $T_{l}, T_{k}$ are variables of $c$, then $S\left(g_{1 j, l k}, g_{1 j, l k}\right)$ reduces to 0 by $g_{j t} \in B$ for $j<t$;

- if $T_{k}$ is a variable of $c$ then $S\left(g_{1 j, l k}, g_{1 t, m k}\right)$ reduces to 0 by $g_{j t}, g_{l m} \in B$ for $j<t, l<m$.

The same argument is applied for the $S$-polynomials of the elements of all $H_{j k_{j}}$ for $j>1$. The assertion follows.

Corollary 1. Let $R=K\left[X_{1}, \ldots, X_{n} ; Y_{1}, \ldots, Y_{n}\right]$ be the polynomial ring over a field $K$ and $L=I_{n-1}+J_{n-1}$.

The presentation ideal of $\operatorname{Sym}_{R}(L)$ admits a lexicographic Gröbner basis of degree $\leq n-1$ in the variables $T_{1}, \ldots, T_{2 n}$.

Corollary 2. Let $R=K\left[X_{1}, \ldots, X_{n} ; Y_{1}, \ldots, Y_{n}\right]$ be the polynomial ring over a field $K$ and $L=I_{n-1}+J_{n-1}$. Then we have: 
1) The presentation ideals of $\operatorname{Sym}\left(I_{n-1}\right)$ and $\operatorname{Sym}\left(J_{n-1}\right)$ have a linear Gröbner basis respectively in the variables $U_{1}, \ldots, U_{n}$ and $V_{1}, \ldots, V_{n}$, which correspond to the generators of $I_{n-1}$ and $J_{n-1}$ respectively.

2) The presentation ideal of $\operatorname{Sym}\left(I_{n-1}+J_{n-1}\right)$ has a Gröbner basis not linear in the variables $T_{1}, \ldots, T_{2 n}$, which correspond to the generators of $L=I_{n-1}+J_{n-1}$.

Proof. 1) Since $I_{n-1}$ and $J_{n-1}$ are generated by an $s$-sequence, there exist a monomial order $\prec_{1}$ in the variables $U_{1}, \ldots, U_{n}$ with $U_{1} \prec_{1} \cdots \prec_{1} U_{n}$ and a monomial order $\prec_{2}$ in the variables $V_{1}, \ldots, V_{n}$ with $V_{1} \prec_{2} \cdots \prec_{2} V_{n}$ such that the presentation ideals of $\operatorname{Sym}\left(I_{n-1}\right)$ and $\operatorname{Sym}\left(J_{n-1}\right)$ have a linear Gröbner basis respectively in the variables $U_{1}, \ldots, U_{n}$ and $V_{1}, \ldots, V_{n}$.

2) It follows by Corollary 1.

\section{STUDYING STANDARD INVARIANTS}

In this section, we shall compute the dimension and the depth of the symmetric algebra $\operatorname{Sym}_{R}(L)$, with $L$ the mixed product ideal $L=I_{n-1}+J_{n-1}$.

In order to apply the theory of $s$-sequences, at the beginning we prove the main result concerning the annihilator ideals of the monomial sequence that generates $L$.

Proposition 1. Let $R=K\left[X_{1}, \ldots, X_{n} ; Y_{1}, \ldots, Y_{n}\right]$ be the polynomial ring over a field $K$ and $L=I_{n-1}+J_{n-1}=\left(f_{1}, \ldots, f_{2 n}\right)$. Then the annihilator ideals of the sequence $f_{1}, \ldots, f_{2 n}$ are:

$$
d_{1}=(0), d_{i}= \begin{cases}\left(X_{n-i+1}\right) & \text { for } i=2, \ldots, n \\ I_{n-1} & \text { for } i=n+1 \\ \left(I_{n-1}, Y_{2 n-i+1}\right) & \text { for } i=n+2, \ldots, 2 n\end{cases}
$$

Proof. Set $f_{i j}=\frac{f_{i}}{\left[f_{i}, f_{j}\right]}$ for $i<j$ and $i, j=1, \ldots, 2 n$. Then the annihilator ideals of the monomial sequence $f_{1}, \ldots, f_{2 n}$ are $d_{i}=\left(f_{1 i}, f_{2 i}, \ldots, f_{i-1, i}\right)$ for $i=$ $1, \ldots, 2 n$. For $i=1$ we have $d_{1}=(0)$ and by the structure of these monomials it follows $d_{2}=\left(f_{12}\right)=\left(X_{n-1}\right), \quad d_{3}=\left(f_{13}, f_{23}\right)=\left(X_{n-2}\right), \ldots, d_{n-1}=$ $\left(f_{1, n-1}, \ldots, f_{n-2, n-1}\right)=\left(X_{2}\right), d_{n}=\left(f_{1 n}, f_{2 n}, \ldots, f_{n-1, n}\right)=\left(X_{1}\right), d_{n+1}=$ $\left(f_{1, n+1}, f_{2, n+1}, \ldots, f_{n, n+1}\right)=\left(f_{1}, \ldots, f_{n}\right)=I_{n-1}, d_{n+2}=\left(f_{1, n+2}, \ldots, f_{n+1, n+2}\right)$ $=\left(f_{1}, \ldots, f_{n}, Y_{n-1}\right)=\left(I_{n-1}, Y_{n-1}\right), \quad \ldots, \quad d_{2 n}=\left(f_{1,2 n}, \ldots, f_{2 n-1,2 n}\right)=$ $\left(f_{1}, \ldots, f_{n}, Y_{1}\right)=\left(I_{n-1}, Y_{1}\right)$. Hence the assertion follows.

Proposition 2. Let $R=K\left[X_{1}, \ldots, X_{n} ; Y_{1}, \ldots, Y_{n}\right]$ be the polynomial ring over a field $K$ and $L=I_{n-1}+J_{n-1}$ for $n \geq 3$. Then

$$
i_{\prec}(J)=\left(d_{2} T_{2}, \ldots, d_{2 n} T_{2 n}\right)+\left(I_{k}^{\prime} X_{n}^{t} T_{1}^{s} T_{j}\right)
$$


where $n+1 \leq j \leq 2 n, 0 \leq k \leq n-3,0 \leq t \leq n-1,0 \leq s \leq n-2$ such that $k+$ $t=n-1, s=t-1$, and $I_{k}^{\prime}$ is the square-free Veronese ideal generated by all the monomials of degree $k$ in the variables $X_{1}, \ldots, X_{n-1}$.

Proof. It is known that the initial ideal of $J$ is given by $\operatorname{in}_{\prec}(J)=\left(\left\{\operatorname{in}_{\prec}(f) \mid f \in\right.\right.$ $B G(J)\})$. One has $B G(J)=\left\{g_{i j}=f_{i j} T_{j}-f_{j i} T_{i}, 1 \leq i<j \leq 2 n\right\} \cup 8$ as in Theorem 3. By the structure of the monomials $f_{1}, \ldots, f_{2 n}$ that generate $L$ we deduce the linear forms $g_{i j}=f_{i j} T_{j}-f_{j i} T_{i}, 1 \leq i<j \leq 2 n$ and then we compute $\operatorname{in}_{\prec}\left(g_{i j}\right)=f_{i j} T_{j}$ for $1 \leq i<j \leq 2 n$ :

$\left\{X_{n-1} T_{2}, \ldots, X_{2} T_{n-1}, X_{1} T_{n}, Y_{n-1} T_{n+2}, \ldots, Y_{1} T_{2 n}, I_{n-1} T_{j}, j=n+1, \ldots, 2 n\right\}$. It remains to compute the initial terms of the element of $\delta$. By the structure of the $S$-polynomials of $\delta$ (see Theorem 3) we obtain that the initial term of these $S$-polynomials are the elements of the set $\left\{I_{k}^{\prime} X_{n}^{t} T_{1}^{s} T_{j}\right\}$, where $I_{k}^{\prime}$ is the ideal generated by the square-free monomials of degree $k$ in the variables $X_{1}, \ldots, X_{n-1}$ and $j=n+1, \ldots, 2 n, k+t=n-1, s=t-1, k=0, \ldots, n-3, t=2, \ldots, n-1$, $s=1, \ldots, n-2$. Then the initial ideal of $J$ is:

$$
\operatorname{in}_{\prec}(J)=\left(X_{n-1} T_{2}, \ldots, X_{1} T_{n}, I_{n-1} T_{n+1},\left(I_{n-1}, Y_{n-1}\right) T_{n+2}, \ldots,\left(I_{n-1}, Y_{1}\right) T_{2 n}\right)
$$
$+\left(I_{k}^{\prime} X_{n}^{t} T_{1}^{s} T_{j}\right)$.

Using Proposition 1 we can write in $\prec(J)=\left(d_{2} T_{2}, \ldots, d_{2 n} T_{2 n}\right)+\left(\left\{I_{k}^{\prime} X_{n}^{t} T_{1}^{s} T_{j} \mid\right.\right.$ $n+1 \leq j \leq 2 n, 0 \leq k \leq n-3,0 \leq t \leq n-1,0 \leq s \leq n-2, k+t=n-1, s=$ $t-1\})$.

Now, we recall some general results about an ideal $I=\left(H_{1} T_{1}, \ldots, H_{t} T_{t}\right) \subset$ $R\left[T_{1}, \ldots, T_{t}\right]$, where $R$ is a noetherian ring and $H_{1}, \ldots, H_{t}$ are ideals of $R$. We say that $I$ is linear in the variables $T_{1}, \ldots, T_{t}$, or $\underline{T}$-linear. For $\underline{T}$-linear ideals we have:

Proposition 3 ([3], Lemma 2.3). Let $I=\left(H_{1} T_{1}, \ldots, H_{t} T_{t}\right)$ be a $\underline{T}$-linear ideal of $R\left[T_{1}, \ldots, T_{t}\right]$. Then

$$
I=\bigcap_{1 \leq r \leq t}\left(H_{i_{1}}+\ldots+H_{i_{r}}, T_{1}, \ldots, \widehat{T}_{i_{1}} \ldots, \widehat{T}_{i_{r}}, \ldots, T_{t}\right),
$$

with $1 \leq i_{1} \leq \ldots \leq i_{r} \leq t$.

Proposition 4 ([3], Prop. 2.4). Let $I=\left(H_{1} T_{1}, \ldots, H_{t} T_{t}\right)$ be a $\underline{T}$-linear ideal of $R\left[T_{1}, \ldots, T_{t}\right]$. Then

$$
d=\operatorname{dim}\left(R\left[T_{1}, \ldots, T_{t}\right] / I\right)=\max _{1 \leq r \leq t}\left\{\operatorname{dim}\left(R /\left(H_{i_{1}}+\ldots+H_{i_{r}}\right)\right)+r\right\}
$$

with $1 \leq i_{1} \leq \ldots \leq i_{r} \leq t$.

In order to apply the previous results, let $\operatorname{in}_{\prec}(J)=\left(d_{2} T_{2}, \ldots, d_{2 n} T_{2 n}\right)+$ $\left(I_{k}^{\prime} X_{n}^{t} T_{1}^{s} T_{j}\right)$ as in Proposition 2. We set $J_{L}=\left(d_{2} T_{2}, \ldots, d_{2 n} T_{2 n}\right)$ the linear part of $\operatorname{in}_{\prec}(J)$ and $J^{*}=\left(I_{k}^{\prime} X_{n}^{t} T_{1}^{s} T_{j}\right)$, where $n+1 \leq j \leq 2 n, 0 \leq k \leq n-3,0 \leq t \leq n-1$, 
$0 \leq s \leq n-2$ such that $k+t=n-1, s=t-1$, and $I_{k}^{\prime}$ is the square-free Veronese ideal generated by all the monomials of degree $k$ in the variables $X_{1}, \ldots, X_{n-1}$.

Proposition 5. Let $R=K\left[X_{1}, \ldots, X_{n} ; Y_{1}, \ldots, Y_{n}\right]$ be the polynomial ring over a field $K, L=I_{n-1}+J_{n-1}$ and $J_{L} \subset R\left[T_{1}, \ldots, T_{2 n}\right]$. Then:

$$
\operatorname{dim}\left(R\left[T_{1}, \ldots, T_{2 n}\right] / J_{L}\right)=2 n+2 .
$$

Proof. Let $J_{L}=\left(d_{1} T_{1}, \ldots, d_{2 n} T_{2 n}\right), \operatorname{dim}\left(R\left[T_{1}, \ldots, T_{2 n}\right] /\left(d_{1} T_{1}, \ldots, d_{2 n} T_{2 n}\right)\right)=$ $\max _{1 \leq r \leq 2 n}\left\{\operatorname{dim}\left(R /\left(d_{i_{1}}+\ldots+d_{i_{r}}\right)\right)+r, 1 \leq i_{1} \leq \ldots \leq i_{r} \leq 2 n\right\}$ by Proposition 3. For $r=1, \ldots, 2 n$ one computes:

$$
\begin{aligned}
& \operatorname{dim}\left(R /\left(d_{i_{1}}+\ldots+d_{i_{r}}\right)\right)+r \leq 2 n+1 \text { for } r<n, \\
& \operatorname{dim}\left(R /\left(d_{i_{1}}+\ldots+d_{i_{r}}\right)\right)+r=[2 n-(n-1)]+n=2 n+1 \text { for } r=n, \\
& \operatorname{dim}\left(R /\left(d_{i_{1}}+\ldots+d_{i_{r}}\right)\right)+r=[2 n-(n-1)]+n+1=2 n+2 \text { for } r=n+1, \\
& \operatorname{dim}\left(R /\left(d_{i_{1}}+\ldots+d_{i_{r}}\right)\right)+r=(2 n-n)+n+2=2 n+2 \text { for } r=n+2, \\
& \operatorname{dim}\left(R /\left(d_{i_{1}}+\ldots+d_{i_{r}}\right)\right)+r=[2 n-(n+1)]+n+3=2 n+2 \text { for } r=n+3,
\end{aligned}
$$

$\operatorname{dim}\left(R /\left(d_{i_{1}}+\ldots+d_{i_{r}}\right)\right)+r=[2 n-(2 n-2)]+2 n=2 n+2$ for $r=2 n$.

Hence $\max _{1 \leq r \leq 2 n}\left\{\operatorname{dim}\left(R /\left(d_{i_{1}}+\ldots+d_{i_{r}}\right)\right)+r\right\}=2 n+2$.

Using the Proposition 5 we state the following

Theorem 4. Let $R=K\left[X_{1}, \ldots, X_{n} ; Y_{1}, \ldots, Y_{n}\right]$ be the polynomial ring over a field $K, L=I_{n-1}+J_{n-1}$ and $J \subset R\left[T_{1}, \ldots, T_{2 n}\right]$ be the relation ideal of $\operatorname{Sym}_{R}(L)$. Then:

1) $\operatorname{dim}\left(R\left[T_{1}, \ldots, T_{2 n}\right] / \operatorname{in}_{\prec}(J)\right)=2 n+1$

2) $\operatorname{depth}\left(R\left[T_{1}, \ldots, T_{2 n}\right] /\right.$ in $\left._{\prec}(J)\right)=2 n+1$

3) $p d\left(R\left[T_{1}, \ldots, T_{2 n}\right] / \operatorname{in}_{\prec}(J)\right)=2 n-1$.

Proof. 1) Let in $\cos _{\prec}(J)=J_{L}+J^{*}$ as in Proposition 2. By the structure of the ideals, $\operatorname{ht}\left(J_{L}+J^{*}\right)=\operatorname{ht}\left(J_{L}\right)+1$. Hence one has

$\operatorname{dim}\left(R\left[T_{1}, \ldots, T_{2 n}\right] / \operatorname{in}_{\prec}(J)\right)=\operatorname{dim}\left(R\left[T_{1}, \ldots, T_{2 n}\right] /\left(J_{L}+J^{*}\right)\right)=$ $\operatorname{dim}\left(R\left[T_{1}, \ldots, T_{2 n}\right]\right)-\operatorname{ht}\left(J_{L}\right)-1=\operatorname{dim}\left(R\left[T_{1}, \ldots, T_{2 n}\right] / J_{L}\right)-1=(2 n+2)-1=$ $2 n+1$ (by Proposition 5). 
2) ht(in $\prec(J))=\operatorname{ht}\left(J_{L}\right)+1=\operatorname{dim}\left(R\left[T_{1}, \ldots, T_{2 n}\right]\right)-\operatorname{dim}\left(R\left[T_{1}, \ldots, T_{2 n}\right] / J_{L}\right)+$ $1=4 n-(2 n+2)+1=2 n-1$. Hence $\operatorname{depth}\left(\operatorname{in}_{\prec}(J)\right) \leq \operatorname{ht}\left(\operatorname{in}_{\prec}(J)\right)=2 n-1$.

By Proposition 2 one has depth(in $\prec(J)) \geq 2 n-1$ being $\left\{X_{n-1} T_{2}, \ldots, X_{1} T_{n}\right.$, $\left.Y_{n-1} T_{n+2}, \ldots, Y_{1} T_{2 n}, I_{k}^{\prime} X_{n}^{t} T_{1}^{s} T_{j}\right\}$ a regular sequence of $2 n-1$ elements of in ${ }_{\prec}(J)$, where $k+t=n-1, s=t-1$ and $n+1 \leq j \leq 2 n$. One has $2 n-1 \leq \operatorname{depth}\left(\operatorname{in}_{\prec}(J)\right) \leq$ $2 n-1$, hence the equality holds. If follows depth(in $\left.\operatorname{in}_{\prec}(J)\right)=\operatorname{ht}\left(\operatorname{in}_{\prec}(J)\right)$, then $\operatorname{in}_{\prec}(J)$ is Cohen-Macaulay that is equivalent to say that $R\left[T_{1}, \ldots, T_{2 n}\right] / \mathrm{in}_{\prec}(J)$ is CohenMacaulay.

Hence $\operatorname{depth}\left(R\left[T_{1}, \ldots, T_{2 n}\right] / \operatorname{in}_{\prec}(J)\right)=\operatorname{dim}\left(R\left[T_{1}, \ldots, T_{2 n}\right] / \operatorname{in}_{\prec}(J)\right)=2 n+1$.

3) $\operatorname{pd}\left(R\left[T_{1}, \ldots, T_{2 n}\right] / \operatorname{in}_{\prec}(J)\right)=\operatorname{dim}\left(R\left[T_{1}, \ldots, T_{2 n}\right]\right)-\operatorname{depth}\left(R\left[T_{1}, \ldots, T_{2 n}\right] /\right.$ $\left.\operatorname{in}_{\prec}(J)\right)=4 n-(2 n+1)=2 n-1$.

The following result holds for the the symmetric algebra $\operatorname{Sym}_{R}(L)$.

Theorem 5. Let $R=K\left[X_{1}, \ldots, X_{n} ; Y_{1}, \ldots, Y_{n}\right], \quad L=I_{n-1}+J_{n-1} \subset R$, $\operatorname{Sym}_{R}(L)=S / J$ with $S=R\left[T_{1}, \ldots, T_{2 n}\right]$.

Then:

1) $\operatorname{dim}\left(\operatorname{Sym}_{R}(L)\right)=2 n+1$

2) $\operatorname{depth}\left(\operatorname{Sym}_{R}(L)\right)=2 n+1$.

3) $p d\left(\operatorname{Sym}_{R}(L)\right)=2 n-1$.

Proof. 1) $\operatorname{dim}\left(\operatorname{Sym}_{R}(L)\right)=\operatorname{dim}\left(S / \operatorname{in}_{\prec}(J)\right)=2 n+1$.

2) One has $\operatorname{depth}\left(\operatorname{Sym}_{R}(L)\right) \geq \operatorname{depth}\left(\operatorname{in}_{\prec}(J)\right)=2 n+1$. On the other hand $\operatorname{depth}\left(\operatorname{Sym}_{R}(L)\right) \leq \operatorname{dim}\left(\operatorname{Sym}_{R}(L)\right)=2 n+1$. The thesis follows.

1.

3) $\operatorname{pd}\left(\operatorname{Sym}_{R}(L)\right)=\operatorname{pd}(S / J)=\operatorname{dim}(S)-\operatorname{depth}(S / J)=4 n-(2 n+1)=2 n-$

Corollary 3. Let $R=K\left[X_{1}, \ldots, X_{n} ; Y_{1}, \ldots, Y_{n}\right], L=I_{n-1}+J_{n-1} \subset R$. Then $\operatorname{Sym}_{R}(L)$ is Cohen-Macaulay.

Example 1. $R=K\left[X_{1}, X_{2}, X_{3} ; Y_{1}, Y_{2}, Y_{3}\right]$

$L=I_{2}+J_{2}=\left(X_{1} X_{2}, X_{1} X_{3}, X_{2} X_{3}, Y_{1} Y_{2}, Y_{1} Y_{3}, Y_{2} Y_{3}\right)$

Set $f_{1}=X_{1} X_{2}, f_{2}=X_{1} X_{3}, f_{3}=X_{2} X_{3}, f_{4}=Y_{1} Y_{2}$,

$f_{5}=Y_{1} Y_{3}, f_{6}=Y_{2} Y_{3}$, where $f_{1} \prec \cdots \prec f_{6}$ with respect to the lex order and $X_{1} \prec X_{2} \prec X_{3} \prec Y_{1} \prec Y_{2} \prec Y_{3}$.

$G=\left\{X_{2} T_{2}-X_{3} T_{1}, X_{1} T_{3}-X_{3} T_{1}, X_{1} X_{2} T_{4}-Y_{1} Y_{2} T_{1}, X_{1} X_{2} T_{5}-Y_{1} Y_{3} T_{1}, X_{1} X_{2} T_{6}-\right.$ $Y_{2} Y_{3} T_{1}, X_{1} T_{3}-X_{2} T_{2}, X_{1} X_{3} T_{4}-Y_{1} Y_{2} T_{2}, X_{1} X_{3} T_{5}-Y_{1} Y_{3} T_{2}, X_{1} X_{3} T_{6}-Y_{2} Y_{3} T_{2}$, $X_{2} X_{3} T_{4}-Y_{1} Y_{2} T_{3}, X_{2} X_{3} T_{5}-Y_{1} Y_{3} T_{3}, X_{2} X_{3} T_{6}-Y_{2} Y_{3} T_{3}, Y_{2} T_{5}-Y_{3} T_{4}, Y_{1} T_{6}-Y_{3} T_{4}$, $\left.Y_{1} T_{6}-Y_{2} T_{5}\right\}$ is a set of generators for $J$. 


$$
B G(J)=G \cup\left\{S\left(g_{13}, g_{24}\right), S\left(g_{13}, g_{25}\right), S\left(g_{13}, g_{26}\right)\right\},
$$

where

$$
\begin{aligned}
& S\left(g_{13}, g_{24}\right)=Y_{1} Y_{2} T_{2} T_{3}-X_{3}^{2} T_{1} T_{4} ; \\
& S\left(g_{13}, g_{25}\right)=Y_{1} Y_{2} T_{2} T_{3}-X_{3}^{2} T_{1} T_{5} ; \\
& S\left(g_{13}, g_{26}\right)=Y_{1} Y_{2} T_{2} T_{3}-X_{3}^{2} T_{1} T_{6} .
\end{aligned}
$$

The annihilator ideals are

$$
\begin{aligned}
& d_{2}=\left(X_{2}\right) ; \\
& d_{3}=\left(X_{1}\right) ; \\
& d_{4}=\left(X_{1} X_{2}, X_{1} X_{3}, X_{2} X_{3}\right)=I_{2} ; \\
& d_{5}=\left(X_{1} X_{2}, X_{1} X_{3}, X_{2} X_{3}, Y_{2}\right)=\left(I_{2}, Y_{2}\right) ; \\
& d_{6}=\left(X_{1} X_{2}, X_{1} X_{3}, X_{2} X_{3}, Y_{1}\right)=\left(I_{2}, Y_{1}\right) .
\end{aligned}
$$

Moreover in $\prec(J)=J_{L}+J^{*}$, with

$J_{L}=\left(\left(X_{2}\right) T_{2},\left(X_{1}\right) T_{3},\left(X_{1} X_{2}, X_{1} X_{3}, X_{2} X_{3}\right) T_{4},\left(X_{1} X_{2}, X_{1} X_{3}, X_{2} X_{3}, Y_{2}\right) T_{5}\right.$, $\left.\left(X_{1} X_{2}, X_{1} X_{3}, X_{2} X_{3}, Y_{1}\right) T_{6}\right)$ and $J^{*}=\left(X_{3}^{2} T_{1} T_{4}, X_{3}^{2} T_{1} T_{5}, X_{3}^{2} T_{1} T_{6}\right)$.

By direct calculations, one obtains:

$$
\begin{aligned}
& \operatorname{dim}\left(\operatorname{Sym}_{R}(L)\right)=\operatorname{depth}\left(S y m_{R}(L)\right)=7 \\
& \operatorname{pd}\left(\operatorname{Sym}_{R}(L)\right)=5 .
\end{aligned}
$$

We are helped by the software $\mathrm{CoCoA}([1])$ for computing examples and formulating results.

\section{ACKNOWLEDGEMENT}

The research that led to the present paper was partially supported by a grant of the group GNSAGA of INdAM.

\section{REFERENCES}

[1] A. Capan, G. Niesi, and L. Robbiano, "Cocoa," A system for doing computations in commutative algebra. Available via anonymous ftp from cocoa.dima.unige.it. 
[2] D. Eisenbud, Commutative Algebra with a view toward algebraic geometry, ser. Graduate Texts in Mathematics, 150. Springer-Verlag, 1995.

[3] J. Herzog, R. G., and Z. Tang, "s-sequences and symmetric algebras," Manuscripta Math., vol. 104, pp. 479-501, 2001, doi: 10.1007/s002290170022.

[4] M. Imbesi and M. La Barbiera, "Invariants of symmetric algebras associated to graphs," Turk. J. Math., vol. 36(3), pp. 345-358, 2012, doi: 10.3906/mat-1010-68.

[5] M. La Barbiera, "On a class of monomial ideals generated by s-sequences," Mathematical Reports, vol. 12(62), pp. 201-216, 2010.

[6] M. La Barbiera and G. Restuccia, "Mixed product ideals generated by s-sequences," Algebra Colloquium, vol. 18(4), pp. 553-570, 2011, doi: 10.1142/\$1005386711000435.

[7] G. Restuccia and V. R.H., "On the normality of monomial ideals of mixed products," Comunications in Algebra, vol. 29(8), pp. 3571-3580, 2001, doi: 10.1081/AGB-100105039.

[8] Z. Tang, "On certain monomial sequences," J. of Algebra, vol. 282, pp. 831-842, 2004, doi: 10.1016/j.jalgebra.2004.08.027.

Authors' addresses

M. La Barbiera

University of Messina, Department of Mathematics, Viale Ferdinando Stagno d'Alcontres, 31, 98166 Messina, Italy

E-mail address: monicalb@unime.it

\section{G. Restuccia}

University of Messina, Department of Mathematics, Viale Ferdinando Stagno d'Alcontres, 31, 98166 Messina, Italy

E-mail address: grest@unime.it 